produced by waves that propagate through the Sun's interior). But when the older (higher) metallicity values are used, the simulations reproduce solar properties very well. This is known as the solar abundance problem, and calls into question the validity of the present models of stellar evolution, or of spectroscopic methods for determining the Sun's composition, or both.

However, the relative contributions of the three different reaction sequences in the $p p$ chain, determined from the Borexino experiment, can be used to infer the temperature in the solar core - a region that is poorly mapped by helioseismological studies. The Borexino findings hint at a core temperature that is consistent with predictions from models that assume high solar metallicity. That said, the results are not yet precise enough to provide a definite answer to the solar abundance problem, because neutrino fluxes predicted by both the high- and low-metallicity solar models are compatible with the new results.

Nevertheless, the Borexino experiment might provide a definite answer in the future. About 1\% of the Sun's nuclear energy is produced through chains of nuclear reactions known as CNO cycles ${ }^{8}$. These cycles are catalysed by the presence of carbon, nitrogen and oxygen, and so their efficiency depends linearly on solar metallicity. If the neutrino fluxes associated with $\mathrm{CNO}$ cycles could be measured, then the abundances of these elements in the solar core could be determined.

Such measurements have proved difficult at Borexino so far, because of background radiation produced by the radioactive decay of bismuth-210 (which forms from the decay of uranium-238, an isotope present in tiny quantities in all matter in the Solar System). Modifications to the vessel that holds the liquid scintillator have now been made ${ }^{9}$ that should address this issue. The detection of CNO neutrinos would not only allow the Sun's metallicity to be determined, but would also provide direct evidence that $\mathrm{CNO}$ cycles occur in nature. This is important, because $\mathrm{CNO}$ cycles are thought to be the main mechanism by which stars more massive than the Sun generate energy ${ }^{8}$.

Another major issue in astrophysics is the proposed existence of non-standard mechanisms for the production or loss of energy in stars ${ }^{10}$. If such a mechanism exists, there will be an imbalance between the solar production rate of nuclear energy and luminosity (the total amount of energy radiated as photons from the Sun's surface). The precision with which the power generated by nuclear reactions in the Sun can be measured would need to be increased tenfold to $1 \%$ to allow tests of such non-standard particle physics. Such precision may be out of reach for Borexino, but it might be possible in future large-scale neutrino and dark-matter detectors.

Aldo Serenelli is in the Astrophysics and Planetary Sciences Department, Institute of Space Sciences (CSIC), and at the

Institut d'Estudis Espacials de Catalunya,

Bellaterra 08193, Spain.

e-mail:aldos@ice.csic.es

1. The Borexino Collaboration. Nature 562, 505-510 (2018).

2. Bergström, J. et al. J. High Energ. Phys. 3, 132 (2016).

3. Mikheyev, S. P. \& Smirnov, A. Y. Yadernaya Fiz. 42, 1441-1448 (1985).

4. Wolfenstein, L. Phys. Rev. D 17, 2369 (1978).
5. Asplund, M., Grevesse, N., Sauval, A. J. \& Scott, P. Annu. Rev. Astron. Astrophys. 47, 481-522 (2009)

6. Grevesse, N. \& Sauval, A. J. Space Sci. Rev. $\mathbf{8 5}$, 161-174 (1998).

7. Basu, S. \& Antia, H. M. Phys. Rep. 457, 217-283 (2008).

8. Haxton, W. C., Hamish Robertson, R. G. \& Serenelli, A. M. Annu. Rev. Astron. Astrophys. 51, 21-61 (2013).

9. Smirnov, O. Data available at https://doi org/10.5281/zenodo.1286860 (2018)

10.Raffelt, G. G. Annu. Rev. Nucl. Particle Sci. 49, 163-216 (1999).

\title{
How to lose your inheritance
}

\section{In developing embryos, molecular and physical differences divide the cells that will form eggs or sperm and those that will form the body. The mouse protein OTX2 directs this decision by blocking reproductive-cell fate.}

\section{DIANA J. LAIRD}

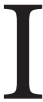
an ultimate act of family planning, the cells destined to contribute to the next generation of an organism are set aside early in embryonic development. It is unclear why primordial germ cells (PGCs), the precursors of eggs and sperm, are established so early in the development of many multicellular organisms. This process of establishing the germ line involves both preventing a non-reproductivecell (somatic-cell) fate and activating a cellular state known as pluripotency - the ability to give rise to the many different cell types in the body. Understanding how the germ line forms is a key requirement for the goal of generating healthy eggs and sperm in vitro for fertility treatment in the clinic. Work in this area has focused mainly on identifying proteins that specify germline fate, but comparatively little is known about why somatic cells do not acquire such a fate. Writing in Nature, Zhang et al. ${ }^{1}$ reveal that the formation of PGCs in mice can be blocked by a protein called OTX2.

In many animals, specification of the germ line operates like a hereditary monarchy in which, like the direct transmission of the crown jewels from one generation to the next, the passage of molecular components in the cytoplasm down the generations determines the cells that will form PGCs. However, some animals, including salamanders, crickets, mice and possibly humans, take a different approach. In the early mouse embryo, designation of the germ line occurs as a result of cells being in the right place at the right time, rather than inheriting the species' crown jewels. In this inductive fate-determination scenario, cells of a cylindrically shaped region of pluripotent cells known as the epiblast are coaxed into adopting a PGC fate by signals from supporting extraembryonic tissues adjacent to the embryo.

In this mode of germline formation, the instructive cues that travel between cells include proteins of the Wnt and BMP families ${ }^{2}$. PGCs normally form at a predictable location in the epiblast (Fig. 1). However, in vivo grafting experiments in mouse embryos revealed that cells from elsewhere in the epiblast have the capacity to become PGCs if they are transplanted to that location ${ }^{3}$. The search for the components that drive germline fate in epiblast cells in response to the 'kingmaker' BMP proteins identified the transcription-factor proteins BLIMP1, PRDM14 and AP2 $\gamma$ (refs 4, 5). This trio of proteins not only drives the expression of genes required to make the germ line, but also blocks the expression of genes associated with a somatic-cell fate $e^{4,5}$.

The process of PGC development can be recapitulated in vitro, starting from mouse embryonic stem (ES) cells that can be induced to form epiblast-like cells. If these epiblastlike cells are exposed to BMPs and certain other factors, then as many as $13.5 \%$ of the cells form PGCs ${ }^{6}$. However, if such epiblastlike cells are engineered to express BLIMP1, PRDM14 and AP2 $\gamma$, more than $30 \%$ form PGCs without the requirement ${ }^{4}$ for BMPs. Yet knowing that a particular pathway can drive the formation of PGCs doesn't answer the questions of whether the default pathway of cellular differentiation in embryonic development is to form germline or somatic cells, or whether all of the cells in the epiblast are equally capable of becoming germline cells. Both matters have implications for our understanding of the evolution of multicellularity, as well as for our ability to generate healthy eggs or sperm from stem cells for clinical applications. 


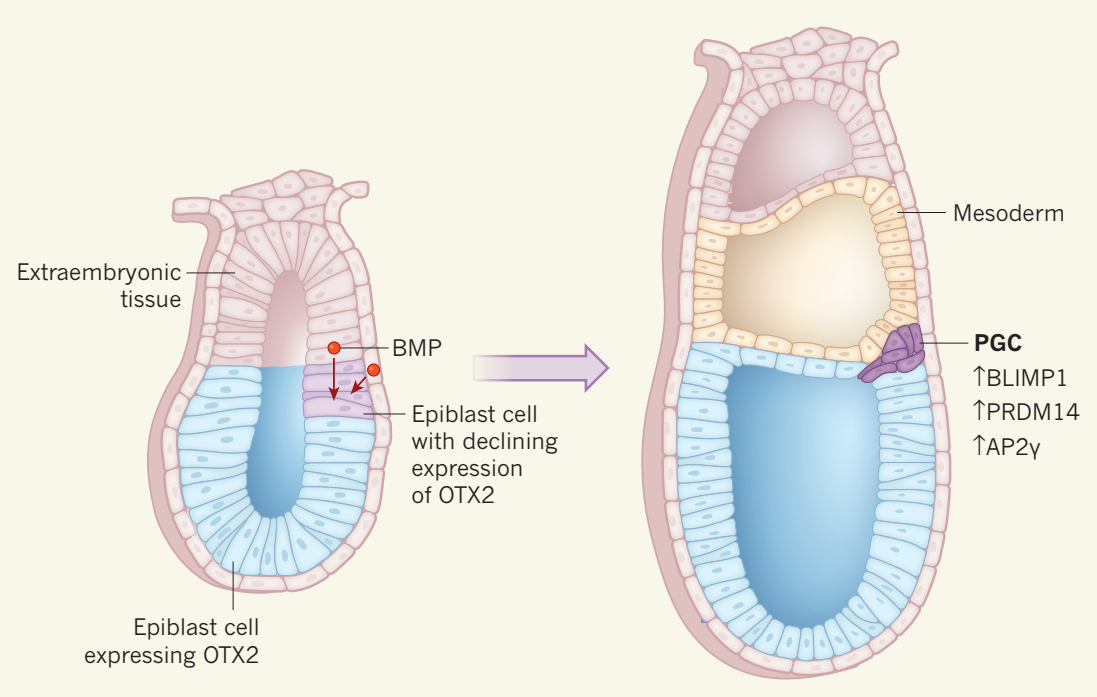

Figure 1 | Expression of the transcription factor OTX2 declines in cells that will form primordial germ cells. Zhang et al. ${ }^{1}$ report how primordial germ cells (PGCs), the precursors of egg and sperm cells, form in vivo in mice from cells in an embryonic region called the epiblast. The formation of PGCs requires a signal from BMP proteins in the adjacent extraembryonic region ${ }^{2}$. The authors report that there is a fall in OTX2 expression in the epiblast region where PGCs will subsequently arise. The drop in OTX2 expression in these cells is followed by a rise in the expression of other transcription factors - BLIMP1, PRDM14 and AP2 $\gamma$ - that are involved the formation of PGCs $s^{4,5}$, which form in an area adjacent to a layer of embryonic cells called the mesoderm. The authors' results indicate that OTX2 blocks the establishment of PGCs.

Zhang and colleagues studied OTX2, a transcription factor known to be involved in the development of the nervous system. In vitro studies had revealed ${ }^{7}$ that OTX2 promotes the transition of mouse ES cells into epiblast-like cells. Zhang et al. scrutinized the expression of OTX2 in mouse stem cells that were differentiating into germline cells in vitro. They found that, in epiblast-like cells progressing towards PGC formation, OTX2 levels decline 12-24 hours before BLIMP1, PRDM14 and AP2 $\gamma$ are upregulated. The authors' in vivo analysis in mice produced similar results (Fig. 1). Considered together, these patterns of expression make sense: the level of OTX2 is low in PGCs and ES cells, both of which exist in a state of pluripotency termed naive pluripotency, which is defined as the ability to give rise to all types of cell in the body. By contrast, the level of OTX2 is high in the epiblast and in ES-cell-derived epiblast-like cells, which are considered to be more restricted in the number of cell lineages they can form.

The timing of the OTX2 decline before the transition from epiblast-cell to PGC fate raises the question of whether OTX2 might prevent cells from transitioning into PGCs. To investigate this, Zhang et al. studied the formation of PGCs in the absence of OTX2. Compared with the situation for wild-type cells, elevated numbers of PGCs were found if embryos lacked the $O t x 2$ gene, or if epiblast-like cells grown in vitro were Otx2-deficient. Conversely, if epiblast-like cells were engineered to express higher than usual levels of OTX2, the formation of PGCs was prevented.

Moreover, the authors found that the acquisition of PGC fate in the absence of Otx 2 could be accomplished in vitro without the usual requirements of BLIMP1 and signalling molecules called cytokines. This was unexpected because in vivo and in vitro experiments ${ }^{8,9}$ have indicated that PGC production requires BLIMP1. How is the requirement for BLIMP1 in germline-cell formation bypassed? Given that OTX2 promotes a form of pluripotency in the epiblast that is more restricted than that of naive pluripotency ${ }^{7}$, perhaps the primary function of BLIMP1 is to create a naive state of pluripotency for newly forming PGCs.

The authors' results are consistent with a model in which BMPs, possibly acting through Wnts, cause the level of OTX2 to decline. This reduction in OTX2 is one of the earliest known steps that determine whether an epiblast cell will acquire a germ-cell or somatic-cell fate. In an intriguing parallel to the system in fruit flies and worms, in which transcription is repressed altogether and cytoplasmic 'crown jewels' are inherited ${ }^{10}$, the first commitment to a PGC fate in mice is now revealed to be a process in which cells are defined by what they are not. Extinguishing the transcription factor OTX2 prevents it from driving gene expression associated with the more-restricted state of pluripotency of the epiblast ${ }^{7}$, instead making way for the naive pluripotency needed for PGCs.

The authors show that the absence of OTX2 drives PGC formation, but it remains to be determined whether these PGCs differentiate normally and form fully functional eggs and sperm. Might the levels of OTX2 affect the fitness of germline cells? For example, would germline cells that have low levels of OTX2 be less likely to successfully contribute to reproduction than cells in which OTX2 has been completely extinguished? Conversely, does the level of OTX2 affect the fitness of somatic cells?

Many other questions remain to be answered. How do BMPs and Wnts cause a decline in OTX2 levels? What is the regulatory relationship between OTX2 and other transcription factors that act in developing PGCs? In the development of the mouse eye ${ }^{11}$, OTX2 drives the expression of BLIMP1 in a process linked to a switch between two types of cell fate, yet in the PGCs, BLIMP1 seems to function after OTX2 levels have declined.

In the developing ear of the fruit fly Drosophila melanogaster ${ }^{12}$, the transcription of OTX2 is directly regulated by the transcription factor N-myc. The known roles of Myc-family genes in metabolism and in a process known as cell competition ${ }^{13}$ suggest that even minor variations in OTX2 levels in the epiblast might reflect a biologically meaningful variation in the fitness of cells that will go on to form the somatic-cell lineages or the germ line. In other words, if it turns out that the levels of Myc correlate with the health of an epiblast cell and its ability to outcompete its neighbouring cells, and if Myc controls the level of OTX2 in mice, then OTX2 might have a role in the fitness of epiblasts, and perhaps in that of PGCs.

OTX2 could provide an avenue for investigating the cell-fate decision between forming germline and somatic cells, and might offer ways of improving PGC differentiation in in vitro approaches. If this mechanism of OTX2-mediated regulation of PGC fate in mice is evolutionarily conserved, then perhaps similar progress might be made in such studies of human cells.

Diana J. Laird is in the Department of Obstetrics, Gynecology and Reproductive Science, University of California, San Francisco, and at the Eli and Edythe Broad Center for Regeneration Medicine and Stem Cell Research, San Francisco, California 94143, USA.

e-mail:diana.laird@ucsf.edu

1. Zhang, J. et al. Nature 562, 595-599 (2018).

2. Ohinata, Y. et al. Cell 137, 571-584 (2009).

3. Tam, P. P. L. \& Zhou, S. X. Dev. Biol. 178, 124-132 (1996).

4. Nakaki, F. et al. Nature 501, 222-226 (2013)

5. Magnúsdóttir, E. et al. Nature Cell Biol. 15, 905-915 (2013).

6. Hayashi, K., Ohta, H., Kurimoto, K., Aramaki, S. \& Saitou, M. Cell 146, 519-532 (2011)

7. Acampora, D., Di Giovannantonio, L. G. \& Simeone, A. Development 140, 43-55 (2013)

8. Ohinata, Y. et al. Nature 436, 207-213 (2005).

9. Vincent, S. D. et al. Development 132, 1315-1325 (2005).

10.Strome, S. \& Lehmann, R. Science 316, 393-393 (2007).

11.Mills, T. S. et al. PLoS ONE 12, e0176905 (2017)

12.Vendrell, V. et al. Development 142, 2792-2800 (2015).

13. Clavería, C. et al. Nature 500, 39-44 (2013). 